\title{
Anti-leucine-rich-glioma-inactivated 1 Limbic Encephalitis: An Underrecognised Disease
}

\author{
Danish Nabi, Mian Ayaz-Ul-Haq, Mehtab Alam, Saqib Ali, Shoaib Ismail and Amjad Iqbal \\ Department of Neurology, Lady Reading Hospital, Peshawar, Pakistan
}

\begin{abstract}
Autoimmune limbic encephalitis is a rare autoimmune disease characterised by inflammation of the limbic system of the brain. The disease runs a sub-acute course with cognitive impairment, memory loss and seizures. These patients have been underdiagnosed in Pakistan. Here, we present a case of a middle-aged male, who presented to our Department after having multiple visits to different psychiatrists for his symptoms. The patient had been put on multiple psychiatric and antiepileptic medications, but his condition gradually declined. At our Department, he was thoroughly assessed and then diagnosed as limbic encephalitis on the basis of the typical history, positive anti-leucine-rich-glioma-inactivated 1 (LGI1) antibodies and MRI findings. The patient responded to plasmapheresis and immune modulating therapy and is being followed up. Emphasis is made on early diagnosis; and earlier treatment of such cases, as it holds a substantial importance in management and makes a difference in future outcome.
\end{abstract}

Key Words: Autoimmune limbic encephalitis, Voltage-gated potassium channels, Cognitive dysfunction.

How to cite this article: Nabi D, Haq MAU, Alam M, Ali S, Ismail S, Iqbal A. Anti-leucine-rich-glioma-inactivated 1 Limbic Encephalitis: An Underrecognised Disease. J Coll Physicians Surg Pak 2021; 31(09):1105-1107.

\section{INTRODUCTION}

Limbic system refers to a collection of brain structures that include the amygdala, hypothalamus, hippocampus, anterior thalamic nuclei, fornix, cingulated gyrus and para-hippocampal gyrus. Limbic encephalitis refers to inflammation of the limbic system, which can be caused by infections, paraneoplastic and autoimmune antibodies. ${ }^{1}$ Leucine-rich-glioma-inactivated 1 (LGII) and contactin-associated protein-like 2 (Caspr2) are two important components of the voltage-gated potassium channels (VGKC)-associated limbic encephalitis. ${ }^{2}$ The main symptoms attributed to the anti-LGI1 antibodies limbic encephalitis are sub-acute cognitive and behavioural impairment, memory decline and seizures. ${ }^{3}$

We report a case of a comparatively rare form of autoimmune encephalitis, the anti-LGI1 antibody limbic encephalitis.

\section{CASE REPORT}

A 40-year male presented with six months' history of progressive cognitive decline, behavioural issues, complex partial seizures, short-term memory and visual hallucinations. The patient had multiple visits to different psychiatrists and was put on different psychiatric and anti-epileptic medications but without much benefit.

Correspondence to: Dr. Danish Nabi, Department of Neurology, Lady Reading Hospital, Peshawar, Pakistan E-mail: danish.nabi01@gmail.com

Received: October 28, 2019; Revised: March 02, 2020;

Accepted: March 14, 2020

DOI: https://doi.org/10.29271/jcpsp.2021.09.1105
The patient arrived at our unit in a drowsy state with a Gasgow coma scale (GCS) of $10 / 15$. The blood tests showed hyponatremia, electoencephalography (EEG) showed encephalopathy, MRI brain showed hyperintensities in the mesial temporal lobes on T2 and FLAIR images (Figure 1). Cerebrospinal fluid (CSF) showed mildly elevated proteins. On suspicion of the typical history of autoimmune encephalitis, the patient was started on high dose of steroids and autoimmune encephalitis antibodies, and screen was sent. The anti-LGI1 antibodies came out to be positive. The treatment choice of intravenous immunoglobulins (IVIGs) or plasmapheresis was discussed with the patients. The attendants opted for plasmapheresis. Five sessions of plasmapheresis were performed on alternate days. The patient responded in the form of mildly improved cognition and was discharged on long-term immunotherapy; and is being followed up.

\section{DISCUSSION}

The incidence of LGI1 encephalitis is reported to be $0.83 / \mathrm{mil}-$ lion/year with elderly males being affected more commonly. ${ }^{4}$ The disease is expected to be underestimated and underdiagnosed. ${ }^{5}$ Limbic encephalitis can becaused by infections as well as autoimmune inflammation. The autoimmune inflammation can be further categorised on the basis of antibodies against the nuclear antigens (paraneoplastic antibodies), antibodies against the target cell surface antigens (VGKC, NMDA) and those associated with unknown antibody (seronegative limbic encephalitis). ${ }^{1,6}$ 


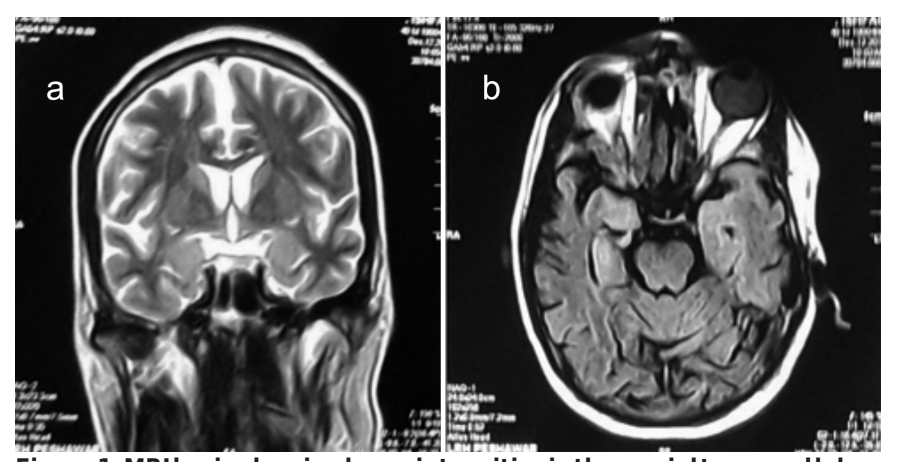

Figure 1: MRI brain showing hyperintensities in the mesial temporal lobes on (a) T2 coronal and (b) FLAIR axial sections.

The VGKCs are situated both in the central nervous system and the peripheral nervous system; and are important regulators of cell action potential. LGI1 and CASPR2 are two of its associated components. ${ }^{4}$ Antibodies against LGI1 were discovered in $2010 .^{4}$ Most of the anti-LGI1 antibodies positive patients present with limbic encephalitis. ${ }^{3}$

LGI1 is a glycoprotein, mainly secreted in the synaptic spaces at the hippocampal and temporal areas where it forms complex with presynaptic VGKCs. ${ }^{4}$ The LGII antibodies were found to disrupt ligand-receptor interaction of LGI1 with ADAM22/23 complex, which results in reversible reduction in synaptic AMPA receptors. ${ }^{7}$

Autoantibody-associated encephalitis, is responsible for a portion of cases of unexplained status epilepticus, encephalitis, and acute-onset psychiatric symptoms. Anti-LGII limbic encephalitis is rarely associated with tumors and the associated tumors are mostly thymomas. ${ }^{2}$

German researchers were the first to report the neuropathological characteristics of anti-LGI limbic encephalitis and suggested a CD8+ T-cell-mediated immune process directed against hippocampal neurons. ${ }^{6}$ Others state that LGII antibodies belong to IgG 4 subclass; and postulated mode of action is through direct functional interference. ${ }^{4}$

The disease has the characteristics of sub-acute memory and cognitive disturbance, behavioural changes, usually accompanied by seizures. ${ }^{3,4,8}$ Three subtypes of seizures can be identified in association with limbic encephalitis: Facio-brachial dystonic seizures (FBDS), subtle autonomic or dyscognitive seizures, and generalised tonic clonic seizures. ${ }^{4,9}$ Recognising FBDS is important as it may be the earliest manifestation of antiLGI1 limbic encephalitis. ${ }^{6}$

The patientcan also have sensory symptoms and auditory hallucination as aura, falls and other seizure semiologies. ${ }^{1}$ Spatial orientation is also affected in these patients. ${ }^{4}$ Few reports have mentioned the presence of reversible insomnia, associated with limbic encephalitis. ${ }^{4,6}$ Autoimmune encephalitis should be considered in patients presenting with sub-acute encephalopathy. ${ }^{10}$

The diagnosis of limbic encephalitis is difficult and often delayed. ${ }^{6}$ Hyponatremia is reported to occur, in about $60 \%$ of patients. ${ }^{2,3}$ Although some lymphocytosis and raised proteins may occur, but usually the routine CSF examination is normal. ${ }^{2}$ MRI and CSF findings may be normal in the early disease course. ${ }^{10}$ About $3 / 4^{\text {th }}$ of patients have a unilateral or bilateral increase in signal intensity in the medial temporal region. One-fourth of the patients may have a normal MRI brain. ${ }^{2,5}$ In the MRI negative patients, brain FDG-PET shows hypermetabolism of the hippocampus, which can help in earlier diagnosis. ${ }^{5}$ EEGs are abnormal but do not have a specific pattern. ${ }^{10}$ The sensitivity of anti-LG1 antibody testing is higher in CSF than in serum.

Early recognition and treatment is vital and is a predictor of better outcome. ${ }^{4,5,10}$ First-line therapy involves high doses of steroids, IVIGs, plasma exchange or mycophenolate. Failure to respond within 1-2 weeks should be treated with 3-5 plasmapheresis sessions or the use of cyclophosphamide or rituximab. ${ }^{4,6,9,10}$ Long-term immunosuppression with rituximab or cyclophosphamide may bring about further neurological improvement. ${ }^{9,10}$

About $70-80 \%$ of the patients show response to treatment with steroids, plasma exchange or immunoglobulins. ${ }^{2}$

Long-term outcome often shows residual deficits. ${ }^{3,4}$ Residual symptoms reported are mostly memory deficits, apathetic behaviour and impaired spatial orientation. Most of the patients had amnesia for the disease period and some retrograde amnesia has been reported as well. ${ }^{3}$

Case-fatality rate at two years follow-up was reported as 19\%. Better recognition of the disease, earlier diagnosis and treatment is expected to decrease the fatality rate. ${ }^{3}$

With better disease recognition, the diagnosis of anti-LGI1 limbic encephalitis is increasing. On suspecting the typical history and presentation of limbic encephalitis, immunotherapy is suggested to be started atonce, even before the antibodies result in the CSF, and blood confirms the disease.

Early recognition of the disease before the development of cognitive impairment, diagnosing the disorder in patients without the criteria of encephalitis, and an aggressive early immunotherapy are important steps towards a good outcome.

\section{PATIENT'S CONSENT:}

Informed consent has been obtained from the patient to publish the case.

\section{CONFLICT OF INTEREST:}

The authors declared no conflict of interest.

\section{AUTHORS' CONTRIBUTION:}

DN: Concept, manuscript writing, data collection, and data analysis.

MAH: Concept, manuscript writing, data analysis and manuscript review.

MA, SI, Al: Manuscriptreview.

SA: Data collection. 


\section{REFERENCES}

1. Gong J, Zhang Y, Wang F, Huang Y, Zhang W. Frequent hemianesthesia as initial symptom of limbic encephalitis associated with LGI1 antibodies. Neurol Sci 2015; 36(10), pp.1953-5. doi: 10.1007/s10072-015-2296-9.

2. Ibrahim $\mathrm{H}, \mathrm{Al}$ Jasser AN, Khan SA, Tlili KG. A rare case of autoimmune limbic encephalitis: An uncharted territory! Neurosciences 2017; 22(4):292-7. doi: 10.17712/nsj. 2017.4.20170150.

3. van Sonderen A, Thijs R, Coenders E, Jiskoot L, Sanchez E, de Bruijn M, et al. Anti-LGI1 encephalitis. Neurol 2016; 87(14):1449-56. doi: 10.1212/WNL.0000000000003173.

4. Bastiaansen AEM, van Sonderen A, Titulaer MJ. Autoimmune encephalitis with anti-leucine-rich gliomainactivated 1 or anti-contactin-associated protein-like 2 antibodies (formerly called voltage-gated potassium channel-complex antibodies). Curr Opin Neurol 2017; 30(3): 302-9. doi: 10.1097/WCO.0000000000000444.

5. Celicanin M, Blaabjerg M, Maersk-Moller C, Beniczky S, Marner L, Thomsen C, et al. Autoimmune encephalitis associated with voltage-gated potassium channels-complex and leucine-rich glioma-inactivated 1 antibodies - a national cohort study. Eur J Neurol 2017; 24(8):999-1005. doi: 10.1111/ene.13324.

6. Liu J, Li M, Li G, Zhou C, Zhang R. Anti-leucine-rich gliomainactivated 1 limbic encephalitis: A case report and literature review. Exp Ther Med 2016; 11(1):315-7. doi: 10.3892/etm.2015.2866.

7. Kurukumbi M, Castillo JA, Shah T, Gupta R. Rare case of anti-LGII limbic encephalitis with new onset epilepsy: $A$ case report. Cureus 2019; 11(5):e4608. doi: 10.7759/ cureus. 4608.

8. Bing-Lei W, Jia-Hua Z, Yan L, Zan Y, Xin B, Jian-Hua S, et al. Three cases of antibody-LGI1 limbic encephalitis and review of literature. Int J Neurosci 2019; 129(7):642-8. doi: 10.1080/00207454.2018.1512985.

9. de Bruijn $M$, van Sonderen A, van Coevorden-Hameete $M H$, Bastiaansen AEM, Schreurs MWJ, Rouhl RPW, et al. Evaluation of seizure treatment in anti-LGI1, anti-NMDAR, and anti-GABABR encephalitis. Neurology 2019; 92(19): e2185-e96. DOI: http://doi.org/10.1212/WNL.00000000 00007475.

10. Mittal MK, Rabinstein AA, Hocker SE, Pittock SJ, Wijdicks EF, McKeon A. Autoimmune encephalitis in the ICU: Analysis of phenotypes, serologic findings, and outcomes. Neurocrit Care 2016; 24(2):240-50. doi: 10.1007/s12028-015 0196-8. 